\title{
IMBEDDING NONDEGENERATE JORDAN ALGEBRAS IN SEMIPRIMITIVE ALGEBRAS
}

\author{
W. S. MARTINDALE III AND K. MCCRIMMON
}

(Communicated by Donald S. Passman)

\begin{abstract}
Zelmanov's structure theory for prime Jordan algebras works directly with semiprimitive algebras, and the results are extended to nondegenerate algebras using properties of the free Jordan algebra. Here we show how Amitsur's direct power trick of imbedding $J$ in the algebra of all sequences from $J$ can be used to imbed any nondegenerate algebra $J$ in a semiprimitive $\bar{J}$ having exactly the same polynomial identities as $J$.
\end{abstract}

Throughout this paper we work with quadratic Jordan algebras $J$ over an arbitrary ring of scalars $\Phi$. Thus $J$ has products

$$
x^{2} \text { and } U_{x} y
$$

quadratic in $x$ and linear in $y$, behaving like the products $x x$ and $x y x$ in associative algebras. We denote the linearization of these products by

$$
x \circ y \text { and }\{x y z\}
$$

(behaving like $x y+y x$ and $x y z+z y x$ ). A Jordan algebra is special if it is isomorphic to a subspace $J \subset A$ of an associative algebra $A$ closed under $x^{2}=x x$ and $U_{x} y=x y x$. Unlike Lie algebras, not all Jordan algebras are special: the archetypal exceptional algebra is the 27-dimensional split albert algebra of hermitian $3 \times 3$ matrices with entries in an octonion or Cayley algebra. Just as we can define a new associative product $a \cdot{ }_{u} b=a u b$, we can define for any $u$ in $J$ a new Jordan algebra, the $u$-homotope

$$
J^{(u)}: \quad x^{(2, u)}=U_{x} u, \quad U_{x}^{(u)} y=U_{x} U_{u} y .
$$

An ideal $I \triangleleft J$ is a subspace invariant under inner and outer multiplication by $J$ $\left(I^{2} \subset I, U_{I} J \subset I\right.$, and $\left.J \circ I \subset I, U_{J} I \subset I\right)$, and we have the usual results about factor algebras $J / I$. We can also create algebras by scalar extension, forming $J_{\Omega}=J \otimes_{\Phi} \Omega$ for any $\Phi$-algebra of scalars $\Omega$. The important special case $\Omega=\Phi[T]$ for a set $T$ of indeterminates leads to the polynomial algebra

$$
J[T]=\left\{\text { all } \sum_{e_{i} \geq 0} x_{e_{1} \cdots e_{n}} t_{1}^{e_{1}} \cdots t_{n}^{e_{n}} \text { for } x_{e_{1} \cdots e_{n}} \in J\right\}
$$

of all formal polynomials in the $t$ 's with coefficients from $J$.

We have notions of prime and primitive Jordan algebras as in the associative theory: an algebra is semiprime or semiprimitive if it is a subdirect product of prime

Received by the editors June 29, 1987.

1980 Mathematics Subject Classification (1985 Revision). Primary 17C10; Secondary 17C20, $17 \mathrm{C65.}$ 
or primitive algebras. More important than semiprimeness is strong semiprimeness or nondegeneracy, the absence of trivial elements $z \neq 0$ with $U_{z}=0$. There are Jordan radicals analogous to their associative counterparts. The Jacobson radical $\operatorname{Rad}(J)$ is the smallest ideal whose factor is semiprimitive. The nil radical $\operatorname{Nil}(J)$ is the largest nil ideal (all of its elements are nilpotent) and the smallest ideal whose factor is nil-free (has no nil ideals). The degenerate (or lower, or McCrimmon) radical $\operatorname{Deg}(J)$ is the smallest ideal whose factor is nondegenerate (this is the "correct" analogue of the semiprime $=$ prime $=$ Baer radical for associative algebras). We always have inclusions

$$
\operatorname{Rad}(J) \supset \operatorname{Nil}(J) \supset \operatorname{Deg}(J)
$$

Zelmanov's Prime Dichotomy Theorem $[1,5.1$, p. 322] asserts that any prime Jordan algebra which can be imbedded in a semiprimitive algebra is either a homomorphic image of a special algebra or is an albert algebra. This applies initially to nil-free-algebras [5] (it can be slightly extended [2] to strictly-nil-free algebras) where one has a semiprimitive scalar extension by

0.5 AMITSUR'S POLYNOMIAL SHRINKAGE [2, 4.1, p. 798, 4.5, p. 799]. The Jacobson radical can be shrunk into the nil radical by polynomial extension: the radical $\operatorname{Rad}(J[t])$ of the polynomial algebra has the form $I[t]$ for an ideal $I$ of $J$ contained in the nil radical $\operatorname{Nil}(J)$. Thus if $J$ has no nil ideals, then $J[t]$ will have no radical ideals.

In [6] the structure theory was extended to nondegenerate algebras using the established structure of the nil-free algebras and the fact that for a universal free algebra the nil and nondegenerate radicals coincide. It is natural to look for a proof which reduces nondegenerate algebras directly to semiprimitive ones, instead of having to pause halfway at the nil-free algebras.

In Zelmanov's Second Dichotomy Theorem [6, 4], describing those prime algebras that are images of special algebras, certain results on clifford identities are established directly for semiprimitive algebras, and must then be extended to nondegenerate algebras. This requires semiprimitive imbeddings which preserve clifford polynomial identities.

These motivate us to look for semiprimitive identity-preserving imbeddings. It was recognized that scalar extensions are too restrictive, but it was asserted $[2, \mathrm{p}$. 806] that "the only general methods known for imbedding a Jordan algebra in a larger one are scalar extension and free product". In this paper we draw attention to a useful imbedding method which was overlooked, despite being well-known in associative P.I. theory (cf. [7, Theorem 1.6.21, p. 45]), namely imbedding $J$ as constant sequences in the sequence algebra or (countable) direct power

$$
\begin{array}{r}
\operatorname{Seq}(J)=\prod_{1}^{\infty} J=\left\{\text { all sequences }\left(x_{1}, x_{2}, \ldots\right) \text { with } x_{i} \in J\right. \\
\text { under componentwise operations }\} .
\end{array}
$$

(This is a special instance of the direct product $\prod_{i \in I} J_{i}$ of algebras under componentwise operations). The map

$$
x \rightarrow(x, x, x, \ldots)
$$


imbeds $J$ in $\operatorname{Seq}(J)$. The point of our paper will be to use sequences to shrink the nil radical into the degenerate radical in the same way that polynomials shrink the Jacobson radical into the nil radical.

1. Identities. In Jordan algebras the associative concept of polynomial identity splits into two concepts. A special or s-identity (s.i.) is a nonzero element $f\left(x_{1}, \ldots, x_{n}\right)$ of the free Jordan algebra $F J(X)$ which vanishes on all special Jordan algebras (equivalently, goes to zero in the free special Jordan algebra $F S J(X)$ under the canonical homomorphism $F J(X) \rightarrow F S J(X))$. An algebra is a homomorphic image of a special algebra iff it satisfies all $s$-identities. A polynomial identity (p.i.) is a nonzero element of the free algebra whose image is nonzero (indeed, has a monic leading term) in $F S J(X)$. A strict identity (str.p.i.) consists of an identity $f\left(x_{1}, \ldots, x_{n}\right)$ together with all its linearizations. We could avoid strict identities by considering only multilinear identities.

We say $J$ satisfies an identity $f$ if $f\left(x_{1}, \ldots, x_{n}\right)$ vanishes under all specializations in $J: f\left(a_{1}, \ldots, a_{n}\right)=0$ for all $a_{i} \in J$. $J$ satisfies a strict identity $f$ ( $f$ and all its linearizations vanish on $J$ ) iff it satisfies $f$ strictly (all scalar extensions $J_{\Omega}$ continue to satisfy $f)$. It is important, but often overlooked, that it suffices if $J[t](\Omega=\Phi[t])$ satisfies $f$ (see $\S 4$ ). If $f$ is multilinear, or $\Phi$ is a field with cardinality greater than the degree of $f$, then $f$ vanishes strictly as soon as it vanishes, but for finite fields or general scalar rings $\Phi$ strictness is not automatic (think of the Boolean condition $\left.x^{2}-x=0\right)$.

In general, if $J$ satisfies an identity (special, polynomial, or strict) so does any subalgebra, homomorphic image, or direct power (e.g. sequence algebra). A scalar extension $J_{\Omega}$ inherits all the strict identities of $J$. If $J^{\prime} \supset J$ inherits all identities (of some sort) for $J$ then so does any image $J^{\prime} / I^{\prime}$, and if $I^{\prime}$ misses $J$ then $J$ remains imbedded in $J^{\prime} / I^{\prime}$ and therefore in turn inherits all identities of this factor algebra.

1.1 PRINCIPLE. If $J^{\prime} \supset J$ inherits all identities of some sort of $J$, and if $I^{\prime} \triangleleft J^{\prime}$ has $I^{\prime} \cap J=0$, then $\bar{J} \supset J$ where $\bar{J}=J^{\prime} / I^{\prime}$ has exactly the same identities of the given sort as $J$. In particular, if $\operatorname{Rad}\left(J^{\prime}\right) \cap J=0$ in this case, then $J$ is imbedded in $\bar{J}=J^{\prime} / \operatorname{Rad}\left(J^{\prime}\right)$ which is semiprimitive and has exactly the same identities of the given sort as $J$.

Our goal is to show that $J^{\prime}=\{\operatorname{Seq}(J[t])\}\left[t^{\prime}\right]$ satisfies this condition for strict identities when $J$ is nondegenerate, and affords the desired imbedding. Note that we cannot remove nondegeneracy here, since if $z \neq 0$ is trivial $\left(U_{z}=0\right)$ then $(z, z, \ldots)$ remains trivial in $J^{\prime}$ and thus $0 \neq z \in J \cap \operatorname{Rad}\left(J^{\prime}\right)$.

2. Direct power shrinkage. We begin by recalling the close connection which Zelmanov first pointed out between degeneracy and elements of bounded index. We say $z$ has strictly bounded index if some fixed power vanishes in all homotopes of all scalar extensions; by (4.3) it suffices if $z^{(n, x)}=0$ for all $x \in J[t]$. This implies $z^{(k, x)} \equiv 0$ for all $k \geq 2 n$, and if $1 / 2 \in \Phi$ for all $k \geq n$. The fundamental connection $[3]$ is

$$
\begin{aligned}
& J \text { is nondegenerate iff it contains no elements } z \neq 0 \text { of strictly } \\
& \text { bounded index. }
\end{aligned}
$$

Just as in associative algebras, there is an easy way to get elements of bounded index. 
2.2 LEMMA. If $z^{2}=z^{3}=(z \circ x)^{n}=0$, then $z^{(2 n+1, x)}=0$, and if $(z \circ x)^{n} \equiv 0$ strictly then $z$ has strictly bounded index.

PROOF. In the case of special algebras

$$
z^{2}=(z \circ x)^{n}=0
$$

implies

$$
0=z(z x+x z)^{n}=z(x z)^{n}=z^{(n+1, x)} .
$$

In the case of general Jordan algebras we need (for the one and only time in the whole paper) some specific identities which hold in all Jordan algebras:

$$
\begin{aligned}
& U_{U_{x} y}=U_{x} U_{y} U_{x} \quad\left(\text { hence } U_{x^{n}}=U_{x}^{n}\right) \\
& U_{x \circ y}+U_{x^{2}, y^{2}}=U_{x} U_{y}+U_{x, y} U_{x, y}+U_{y} U_{x} \\
& \{x y y\}=x \circ y^{2} \\
& U_{x, z} U_{z}=V_{x, z} V_{z^{2}}-V_{x, z^{3}}
\end{aligned}
$$

where $U_{x, z} y=\{x y z\}=V_{x, y} z$. In general, if $z^{2}=z^{3}=(z \circ x)^{n}=0$ then $0=$ $U_{(z \circ x)^{n}} z=U_{z \circ x}^{n} z($ by $(2.3 \mathrm{a}))=\left\{U_{z} U_{x}+U_{x} U_{z}+U_{z, x}^{2}-U_{z^{2}, x^{2}}\right\}^{n} z$ (by (2.3b)) $=\left(U_{z} U_{x}\right)^{n} z$ (using $z^{2}=0, U_{z} z=z^{3}=0, U_{z, x} z=z^{2} \circ x=0$ (by (2.3c)), and repeatedly using $U_{z} U_{z}=U_{z^{2}}=0$ by (2.3a), $U_{z, x} U_{z}=V_{x, z} V_{z^{2}}-V_{x, z^{3}}=0$ (by $(2.2 \mathrm{~d}))$ ), where $\left(U_{z} U_{x}\right)^{n} z=U_{z}^{(x) n} z=z^{(2 n+1, x)}$ in the homotope $J^{(x)}$ of $(0.2)$. If $(z \circ x)^{n}$ vanishes strictly, then so does $z^{(2 n+1, x)}$.

The key idea is that for a sequence to be nilpotent, all its terms must be nilpotent and there must be a bound on the indices of nilpotence.

2.4 AMITSUR'S DIRECT-POWER TRICK. If $z \in J \cap \operatorname{Nil}(\operatorname{Seq}(J))$ then there is an integer $n=n(z)$ such that $(z \circ x)^{n}=0$ for all $x \in J$.

PROOF. If no $n$ works for all $x$ at once, there exist $x_{n} \in J$ with $\left(z \circ x_{n}\right)^{n} \neq 0$, and the element $(z, z, \ldots) \circ\left(x_{1}, x_{2}, \ldots\right)=\left(z \circ x_{1}, z \circ x_{2}, \ldots\right)$ in $\operatorname{Seq}(J)$ is not nilpotent of any finite index, so $(z, z, \ldots)$ does not belong to $\operatorname{Nil}(\operatorname{Seq}(J))$.

2.5 A MITSUR'S DIRECT-POWER SHRINKAGE. $J \cap \operatorname{Nil}(\operatorname{Seq}(J[t]))$ is an ideal of $J$ contained in $\operatorname{Deg}(J)$.

ProOF. $I=J \cap \operatorname{Nil}(\operatorname{Seq}(J[t]))$ is certainly an ideal in $J$, and to show it is contained in $\operatorname{Deg}(J)$ it suffices to show $\bar{I}$ vanishes in $\bar{J}=J / \operatorname{Deg}(J) \subset$ $\operatorname{Seq}(J[t]) / \operatorname{Seq}(\operatorname{Deg}(J)[t]) \cong \operatorname{Seq}(\bar{J}[t])$. Now $\bar{I}$ still lies in the nil radical Nil $(\operatorname{Seq}(\bar{J}[t]))$ of the factor algebra, so (as usual for radical surgery) it suffices to prove $\bar{I}=$ $\bar{J} \cap \operatorname{Nil}(\operatorname{Seq}(\bar{J}[t]))=\overline{0}$ for nondegenerate $\bar{J}$. But if $\bar{I} \neq \overline{0}$ there is $\bar{z} \neq \overline{0}$ in $\bar{I}$ with $\bar{z}^{2}=\bar{z}^{3}=\overline{0}$, and by (2.4) applied to $\bar{J}[t]$ for some $n(\bar{z} \circ \bar{x})^{n}=\overline{0}$ for all $\bar{x} \in \bar{J}[t]$, therefore $(\bar{z} \circ \bar{x})^{n}=\overline{0}$ strictly as a function of $\bar{x} \in \bar{J}$ by 4.3 , so by Lemma $2.2 \bar{z}$ has strictly bounded index, which by $(2.1)$ contradicts nondegeneracy of $\bar{J}$. Thus $\bar{I}=\overline{0}$ and $I \subset \operatorname{Deg}(J)$.

It is not clear whether in fact the intersection is exactly $\operatorname{Deg}(J)$, equivalently whether $\operatorname{Deg}\left(J^{\prime}\right) \subset \operatorname{Nil}\left(\operatorname{Seq}\left(J^{\prime}\right)\right)$ for all $J^{\prime}$. 
3. The main imbedding theorem. We are now ready to combine polynomial and sequential shrinkage to obtain the

3.1 NONDEGENERATE IMBEDDING THEOREM. If $J$ is a nondegenerate Jordan algebra, then $J$ may be imbedded in a semiprimitive algebra $\bar{J}$ in such a way that $\bar{J}$ satisfies exactly the same strict identities as $J$.

Proof. We have $J=J_{0} \subset J_{1} \subset J_{2} \subset J_{3}=J^{\prime}$ for $J_{1}=J[t], J_{2}=\operatorname{Seq}\left(J_{1}\right)=$ $\operatorname{Seq}(J[t])$, and $J_{3}=J_{2}\left[t^{\prime}\right]=\{\operatorname{Seq}(J[t])\}\left[t^{\prime}\right]$. Here each $J_{i+1}$ inherits nondegeneracy and all strict identities from $J_{i}$ (a sequence is trivial iff all its terms are, a polynomial is trivial only if its top degree term is). By our general Principle 1.1 we need only show that

$$
J \cap \operatorname{Rad}\left(J^{\prime}\right)=0
$$

to get the desired imbedding $J \subset \bar{J}=J^{\prime} / \operatorname{Rad}\left(J^{\prime}\right)$. But by Polynomial Shrinkage $0.6 J \cap \operatorname{Rad}\left(J^{\prime}\right)=J \cap J_{2} \cap \operatorname{Rad}\left(J_{2}\left[t^{\prime}\right]\right) \subset J \cap N i l\left(J_{2}\right)$, and by Direct-Power Shrinkage $2.5 J \cap \operatorname{Nil}\left(J_{2}\right)=J \cap \operatorname{Nil}(\operatorname{Seq}(J[t])) \subset \operatorname{Deg}(J)=0$ by nondegeneracy, establishing (*).

Zelmanov's Prime Dichotomy Theorem is proven under the explicit hypothesis that the prime algebra is imbeddable in a semiprimitive algebra, so from 3.1 without further ado or polynomial identities we have

3.2 PRIME DiChOTOMY THEOREM. Any nondegenerate prime Jordan algebra is either a homomorphic image of a special algebra, or is an albert algebra.

In a forthcoming paper [4] it will be important that the semiprimitive imbeddings can be chosen to preserve polynomial identities.

4. Appendix on polynomial mappings. To avoid multi-indices and simplify notation, we pass from several variables to a single vector variable, i.e. from maps $J^{n} \stackrel{f\left(x_{1}, \ldots, x_{n}\right)}{\longrightarrow} J$ to the general case of abstract polynomial mappings $X \stackrel{f(x)}{\longrightarrow} Y$ of $\Phi$-modules in the sense of Robi [9] (cf. [8, pp. 202-207]). We say such a map $f$ vanishes strictly on $X$,

$$
f \equiv 0 \quad \text { on } X
$$

if $f$ vanishes on all scalar extensions $X_{\Omega}$. Strictness is equivalent to $f$ vanishing on the particular "universal" extension $X\left[t_{1}, t_{2}, \ldots\right]$ for an infinite set of indeterminates, and also equivalent to the intrinsic condition that all linearizations of $f$ vanish on $X$. Here the linearizations $f_{e_{1}, \ldots, e_{r}}\left(x_{1}, \ldots, x_{r}\right)$ of $f$ are defined as the coefficients of powers of $t$ 's in the expansion

$$
f\left(t_{1} x_{1}+\cdots+t_{r} x_{r}\right)=\sum_{e_{1}+\cdots+e_{r} \leq d} t_{1}^{e_{1}} \cdots t_{r}^{e_{r}} f_{e_{1} \cdots e_{r}}\left(x_{1}, \ldots, x_{r}\right)
$$

for $d$ the degree of $f, x_{i}$ in $X$. (This is the place where multi-indices would be awkward, if we had to linearize each variable $x_{i}$ in $f\left(x_{1}, \ldots, x_{n}\right)$.)

It is often useful to get by with a single $t$.

4.3 ONE- $t$-IS-ENOUGH LEMMA. If $f: X \rightarrow Y$ is a polynomial mapping whose extension to $X[t] \rightarrow Y[t]$ vanishes, then $f$ vanishes strictly on $X$. 
ProOF. The hypothesis that $f$ vanishes on $X[t]$ means for all $x_{1}, \ldots, x_{r}$ in $X$ and any choice of integral powers $a_{1}, \ldots, a_{r}$ the value

$$
f\left(t^{a_{1}} x_{1}+\cdots+t^{a_{r}} x_{r}\right)=\sum t^{a_{1} e_{1}+\cdots+a_{r} e_{r}} f_{e_{1} \cdots e_{r}}\left(x_{1}, \ldots, x_{r}\right)
$$

is zero in $Y[t]$, i.e., the coefficient of $t^{k}$ vanishes in $Y$ for each $k$. To conclude strictness $f_{e_{1} \cdots e_{r}}\left(x_{1}, \ldots, x_{r}\right)=0$ for each $r$-tuple $\mathbf{e}=\left(e_{1}, \ldots, e_{r}\right)$ in $N^{r}\left(\sum e_{i} \leq d\right)$ and each $r$-tuple $\left(x_{1}, \ldots, x_{r}\right)$ in $X^{r}$, we need only show that we can choose $\mathbf{a}=$ $\left(a_{1}, \ldots, a_{r}\right)$ so that the $\mathbf{a} \cdot \mathbf{e}$ are all distinct, i.e. $\mathbf{a} \cdot\left(\mathbf{e}-\mathbf{e}^{\prime}\right) \neq 0$ for $\mathbf{e} \neq \mathbf{e}^{\prime}$. But there are at most $d^{r}$ such $n$-tuples $e$, hence at most $k=d^{r}\left(d^{r}-1\right)$ such $\mathbf{e}-\mathbf{e}^{\prime}$, and given any finite number $\mathbf{v}_{1}, \ldots, \mathbf{v}_{k}$ of nonzero vectors in $Q^{r}$ we can find $\mathbf{a} \in N^{r}$ with $\mathbf{a} \cdot \mathbf{v}_{i} \neq 0$ for all $i=1,2, \ldots, k$. One way is to note $f_{i}(\mathbf{a})=\mathbf{a} \cdot \mathbf{v}_{i}$ is a nonzero linear function from $Q^{r}$ to $Q$ if $\mathbf{v}_{i} \neq 0$, so the pointwise product $f=f_{1} \cdots f_{k}$ and the function $g(a)=f(\operatorname{sq}(\mathbf{a}))$ (where $\operatorname{sq}(\mathbf{a})=\left(a_{1}^{2}, \ldots, a_{r}^{2}\right)$ is the componentwise square) are not identically zero as functions $Q^{r} \rightarrow Q$ since the field $Q$ is infinite, and if $g$ does not vanish at $\mathbf{b}$ then by homogeneity we can clear denominators to get $g(\mathbf{b}) \neq 0$ for $\mathbf{b} \in Z^{r}$, so $f(\mathbf{a}) \neq 0$ for $\mathbf{a}=\mathrm{sq}(\mathbf{b}) \in N^{r}$, therefore no $f_{i}(\mathbf{a})$ vanishes, and $\mathbf{a} \cdot \mathbf{v}_{i} \neq 0$ for all $i$.

Alternately, we can "explicitly" exhibit $\mathbf{a} \in N^{r}$ with $\mathbf{a} \cdot \mathbf{v}_{i} \neq 0$ for any given finite set $\mathbf{v}_{1}, \ldots, \mathbf{v}_{k}$ of nonzero vectors in $Z^{r}$. Indeed, let $p_{1}, \ldots, p_{r}$ be distinct prime integers such that $p_{j}$ does not divide any nonzero $j$ th entry (if $\mathbf{v}_{i}=\left(b_{i 1}, \ldots, b_{i r}\right)$ then $p_{j}$ does not divide any of $b_{1 j}, \ldots, b_{k j}$ which are nonzero; if all $j$ th entries $b_{i j}$ are zero we agree $\left.p_{j}=1\right)$, and set $\mathbf{a}=\left(a_{1}, \ldots, a_{r}\right)$ for $a_{i}=\prod_{l \neq i} p_{l}$. Since the vector $\mathbf{v}_{i}$ is nonzero, at least one of its entries $b_{i j}$ is nonzero, hence by construction $p_{j}$ does not divide $b_{i j}$ or $a_{j}$ yet divides all other $a_{l}$, so $p_{j}$ does not divide $\mathbf{a} \cdot \mathbf{v}_{i}=$ $a_{1} b_{i 1}+\cdots+a_{j} b_{i j}+\cdots+a_{r} b_{i r}$ and therefore this latter cannot be zero.

ACKNOWLEDGEMENT. The authors would like to thank NSF Grant DMS8401066 for partially supporting this research.

\section{REFERENCES}

1. K. McCrimmon, Zelmanov's prime theorem for quadratic Jordan algebras, J. Algebra 76 (1982), 297-326.

2. __ Amitsur shrinkage of Jordan radicals, Comm. Algebra 12 (1984), 777-826.

3. $\ldots$ A characterization of the nondegenerate radical in quadratic Jordan triple systems, Algebras Groups Geom. 4 (1987), 145-164.

4. K. McCrimmon and E. Zelmanov, The structure of strongly prime quadratic Jordan algebras, Adv. in Math. (to appear).

5. E. Zelmanov, On prime Jordan algebras, Algebra i Logika 18 (1979), 162-175.

6. __ On prime Jordan algebras. II, Siberian Math. J. 24 (1983), 89-104.

7. L. Rowen, Polynomial identities in ring theory, Academic Press, New York, 1980.

8. O. Loos, Jordan pairs, Lecture Notes in Math., vol. 460, Springer-Verlag, Berlin and New York, 1975.

9. N. Robi, Lois pôlynomes et lois formelles en theorie de modules, Ann. Sci. Ecole Norm. Sup. $3^{\mathbf{e}}$ Ser. 80 (1963), 213-318.

Department of Mathematics, University of Massachusetts, Amherst, MASSACHUSETTS 01033

Department of Mathematics, University of Virginia, Charlottesville, VIRGINIA 22903 